\title{
Cómo la tradición continental y la tradición analítica se enfrentan con la tradición filosófica ${ }^{1}$
}

\section{(How Do Continental and Analytic Traditions Confront the Philosophical Tradition)}

\author{
François JARAN \\ Universidad de Valencia \\ francois.jaran@uv.es
}

Recibido: 6 de mayo de 2011

Aceptado: 7 de septiembre de 2011

\section{Resumen}

La relación que mantiene la filosofía presente con la filosofía del pasado es un tema fundamental para entender la división actual entre la filosofía analítica y la filosofía continental. Sin embargo, la oposición no se sitúa en el mero rechazo o la aceptación de la historia de la filosofía. De hecho, las dos tradiciones filosóficas contemplan la posibilidad de un dialogo con los grandes filósofos del pasado. Este estudio caracteriza la relación con el pasado propia de cada tradición y termina argumentando a favor de la relevancia de la historia de la filosofía en filosofía.

Palabras claves: Filosofía continental, filosofía analítica, filosofía de la historia, exactitud histórica, historicismo.

\begin{abstract}
The relationship between present-day philosophy and philosophy of the past is a fundamental issue for understanding today's philosophical division between

1 Este trabajo ha recibido el apoyo de la Alexander von Humboldt-Stiftung y del Ministerio de Ciencia e Innovación (Plan nacional I+D, FFI-2009-11921).
\end{abstract}


"analytical" and "continental" philosophy. However, the opposition doesn't lie in the mere rejection or acceptation of philosophy's history. In fact, both philosophical traditions conceive the possibility of a dialog with the great philosophers of the past. This paper first characterizes the relationships with past philosophy in both traditions and arguments in favor of the relevancy of philosophy's history for philosophy.

Keywords: Continental Philosophy, Analytic Philosophy, Philosophy of History, Historical accuracy, Historicism.

\section{Introducción}

El tema de este breve estudio es el de la relación entre la filosofía y su historia. No entre filosofía e historia, sino entre filosofía e historia de la filosofía. Según una idea muy extendida, el filósofo y el historiador de la filosofía practican oficios distintos que corresponden a tareas distintas pero no incompatibles. La cuestión que abordaré aquí es si no son algo más que compatibles, es decir, si no tienen que ser complementarias o hasta necesitarse la una de la otra.

En la medida en que, para muchos, esta cuestión es precisamente la que divide hoy en día la filosofía en dos campos opuestos -llamados, por motivos poco filosóficos "analítico" y "continental"2 - la pregunta sobre la relación entre filosofía e historia de la filosofía debería cobrar una importancia enorme en el panorama filosófico actual. Quiero aportar una pequeña contribución a esa pregunta gigantesca tratando de arrojar luz a la cuestión de la posibilidad del diálogo entre filósofos del presente y filósofos del pasado. En este marco, me interesarán los puntos de encuentro y de desencuentro de las dos tradiciones.

En los últimos treinta años se han dedicado varias obras colectivas a este tema, tanto en la tradición analítica como en la tradición continental ${ }^{3}$. Esos debates sobre la relación entre filosofía e historia de la filosofía ponen de manifiesto, entre otras cosas, la falsedad de los prejuicios que suele tener cada tradición sobre la otra: que la filosofía analítica ignora por completo el pasado de la filosofía o que la filosofía continental es incapaz de distinguir entre filosofía e historia de la filosofía. Es cierto que las tradiciones se distinguen en cuanto a su relación con la historia, pero las cosas no se dejan caracterizar de forma tan sencilla. Podemos añadir que aunque esta cuestión divida las dos corrientes filosóficas, constituye sin embargo uno de los

\footnotetext{
2 Sobre el origen de esas dos expresiones, véanse Rée (1997) y Grondin (2000). Para una presentación general de las dos “escuelas”, véanse D’agostini (1997) y Sáez Rueda (2002).

${ }^{3}$ Entre los más importantes: Sorell y Rogers (2005), Schuback y Ruin (2005), Courtine (1999), Vienne (1997), Boss (1994), Vattimo (1989), Hare (1988), Rorty, Schneewind y Skinner (1984).
} 
pocos temas donde pueda existir un debate fecundo entre las dos tradiciones. Cabe señalar que en la mayoría de los casos, la oposición entre las dos formas filosóficas que conocemos no se debe a que cada una adopte una posición opuesta sobre un mismo problema, sino más bien a que casi no existan problemas que les sean comunes. Intentaré aquí caracterizar las formas que cobran los diálogos con la historia de la filosofía en las dos tradiciones para dar algunas pistas sobre la importancia que tiene, a mi modo de ver, la historia de la filosofía para la filosofía. Pero para empezar, voy a tratar de caracterizar varios modelos de historia para después hablar de cómo se ha relacionado, a lo largo de la historia, la filosofía con su propia historia.

\section{Modelos de historia}

La filosofía, en cuanto ciencia, mantiene una relación peculiar con su historia. Al contrario de otros modos de conocimiento, le suele dar una importancia -desmesurada para muchos- a su historia. En ninguna facultad universitaria se habla tanto de la historia propia. Es cierto que los físicos han oído hablar de Newton, pero a ninguno se le ha exigido la lectura de los Philosophiae naturalis principia mathemati$c a$. Para ellos, eso no supone ninguna limitación profesional, como mucho una laguna en su formación cultural (Sánfelix, 2007, p. 474). Aunque la historia sirva a menudo de introducción didáctica a las ciencias, el pasado de la ciencia no es considerado una fuente de conocimiento y suele presentarse del mismo modo que se presenta a Lamarck antes que Darwin, o sea, como concepciones contradichas y superadas por teorías más recientes. A pesar de reconocer su deuda con el pasado, la ciencia se presenta como un progreso continúo que no sabe avanzar sin transformar en obsoletas las posiciones anteriores.

Si en el marco de su práctica, los científicos no se interesan por su propia historia, eso se debe a que la "historicidad" de sus conceptos no afecta en absoluto la comprensión de la ciencia misma 4 . Eso se debe, en palabras de Bernard Williams, a que la ciencia tiene una "historia vindicatoria". En el curso de la historia de la ciencia, las teorías ulteriores no sólo se justifican ellas mismas, sino que también explican el modo de ver propio de las teorías anteriores y justifican la transición entre la una y la otra. De este modo, tanto los defensores de la teoría anterior como los de la teoría ulterior deben reconocer en esta transición un progreso (Williams, 2000, 486).

Esta relación con el pasado se asemeja a la que tienen los saberes técnicos o la

\footnotetext{
${ }^{4}$ Por ciencia, entendemos aquí el trabajo que se lleva a cabo en campos como la biología y química e imaginamos al científico trabajando en investigaciones concretas (en un laboratorio, por ejemplo) y no tanto en investigaciones fundamentales acerca de las definiciones de conceptos y demás. No pensamos en ciencias como las matemáticas o la física fundamental cuyas relaciones con la historia son quizás tan problemáticas como las que unen la filosofía a la historia.
} 
artesanía con su historia. Los carpinteros, por ejemplo, poseen técnicas que han tardado varios milenios en perfeccionarse. Las técnicas actuales tienen, sin embargo, poco que ver con las antiguas y el conocimiento de la historia de la carpintería y de su trasmisión no le es de ninguna utilidad al carpintero para llevar a cabo su tarea. Es cierto que, en la medida en que el carpintero usa técnicas llegadas a un estadio preciso de su desarrollo, podemos decir que su trabajo se inscribe en la historia, pero no de forma esencial. En la práctica concreta de una técnica, el conocimiento explícito de la historia de la técnica no tiene ninguna consecuencia. Este conocimiento tendría importancia sólo en la medida en que el carpintero quiera también ser creador en el sentido artístico de la palabra.

A diferencia de las ciencias y de los saberes técnicos, las artes mantienen una relación bien distinta con su historia. Eso puede deberse sin duda a que no entretienen un sentimiento de superioridad con el pasado artístico, al contrario de las ciencias y de las técnicas. Las épocas artísticas no se suceden dejando obsoletas a las anteriores, sino en un nivel meramente material. A ese nivel, el artista se encuentra de hecho en la situación del artesano o del técnico que opera con las técnicas disponibles en su época y se inscribe también en la historia, pero no de forma esencial. El pintor pinta con pinceles y pinturas propios de su tiempo y depende, en ese sentido de una cierta evolución de las técnicas artísticas. Sin embargo, lo que distingue la relación del artesano y la del artista con sus historias respectivas es la parte creadora de su trabajo, ya sea la elección de los temas, el modo de representarlos, etc. El artista no puede crear sin hacer referencia explícita o no a la historia del arte. Al pintar, el pintor se inscribe siempre en la historia de la pintura aunque su conocimiento de ella sea limitado. Cada artista está obligado a tomar posición frente a la tradición que le precede, bien sea positiva o negativamente. El sentido de una obra de arte o de un avance artístico no se puede entender sin el contexto en el que se presenta. La supresión de la línea en el impresionismo, por ejemplo, no tendría ninguna significación si la tradición que le ha precedido no hubiera usado la línea como el fundamento del dibujo. Del mismo modo, la música dodecafónica cobra sentido en su diferencia con la música clásica. En la práctica concreta de su arte, el artista pone de manifiesto la tradición histórica a partir de la cual está creando. Los conceptos de canon y de clásico se han usado en historia del arte para explicitar ésta inscripción esencial del artista en la historia del arte, inscripción que no es equiparable con la del técnico y la del científico.

Esto no significa, sin embargo, que el arte y la historia del arte sean el mismo oficio. Existe de hecho una distinción bien clara entre, por un lado, el trabajo del artista y las dotes que tiene que poseer para llevarlo a cabo y, por el otro, el trabajo del historiador del arte y sus propias dotes. Aunque para el artista sea esencial esa relación con la tradición, no considera la tarea del historiador como propia de su trabajo. Además, el conocimiento de la historia del arte que tenga un artista es distin- 
ta de la que tiene el historiador. Quizás sea a menudo fragmentaria y a veces inexacta, pero se trata de un saber mucho más directo que el del historiador, en la medida en que el artista reconoce en el trabajo artístico de otros artistas algo que el historiador sólo puede adivinar: el trabajo realizado, el intento de adecuarse a lo representado, etc.

Para presentar las relaciones artísticas y técnico-científicas con la historia, podemos decir que a pesar de que los dos campos estén inscritos en la historia, sólo en el arte se establece un verdadero diálogo con la historia. La ciencia está obviamente determinada por su pasado, pero no en cuanto pasado. Las leyes, los teoremas y los modelos pasados y que siguen vigentes son los únicos que importan al científico. Y no le importan en cuanto herencia, sino en cuanto posesión presente de un conocimiento. Es la validez presente de sus "doctrinas" lo que hace que figuras científicas históricas puedan ser relevantes.

Las cosas no funcionan así en arte en la medida en que nada tiene "validez" como tal. Si en ciencia se puede siempre reactivar una demostración y justificar debidamente el uso, por ejemplo, de una ley física, en arte no se puede porque la "validez" de los clásicos no consiste en adecuarse a nuestros criterios estéticos actuales, sino, al contrario, en haberse impuesto a nuestra "conciencia estética", por ser ellos mismos los que han establecido nuestros criterios.

$\mathrm{El}$ arte no tiene un mundo natural al que adecuarse y frente al cual comprobar la validez de sus realizaciones. Una obra de arte conseguida se adecua no tanto a la naturaleza como a la historia, es decir, a un ámbito algo más secreto y cuyos acontecimientos no siguen ninguna ley cognoscible y no son por tanto previsibles.

Pero nuestra pregunta no versa sobre arte o ciencia, sino sobre filosofía: ¿qué modelo debe seguir la filosofía? ¿cuál de los distintos modelos corresponde mejor a la esencia propia de la filosofía? Obviamente, la antigüedad de la filosofía le exime de someterse a cualquiera de los modelos que aquí hemos presentado. Sin embargo, pueden servir -y de hecho sirven- de referencia para interrogar la relación que tiene con su historia 5 .

Para muchos, al igual que con la tradición artística, la historia de la filosofía sigue siendo la mejor escuela para el aprendizaje del oficio de filósofo. Al leer textos históricos de filosofía, hacemos algo que el físico no puede hacer leyendo a Newton y que el artista sí hace estudiando artistas antiguos: aprender un oficio. Porque por muy imperfectas que sean las filosofías antiguas, nos permiten, como decía Kant, quizás no aprender la filosofía, pero sí aprender a filosofar. En ese sentido, el filósofo podría considerar su relación con la historia como muy cercana a la del artista. La filosofía tiene sus clásicos y, en cierta medida, sus cánones, y nunca

\footnotetext{
5 Por falta de competencia, dejamos aquí voluntariamente de lado la relación que existe entre la religión y su pasado, así como la que liga la teología a la historia de la teología. Pero a diferencia de la filosofía, en estos dos casos, no se contempla la posibilidad de dejar atrás a la tradición o de negar la vinculación esencial a acontecimientos pasados.
} 
avanza una tesis sin poner de manifiesto, explícita o implícitamente, voluntaria o involuntariamente, la tradición histórica desde la cual está trabajando. Esto se nota en la elección de los conceptos, de los problemas y de los métodos. Siguiendo el modelo de la historia del arte, cabría añadir que la calidad del trabajo filosófico no depende, sin embargo, de la exactitud del conocimiento histórico que el filósofo tenga.

Si ese modelo no obtiene el consenso entre los filósofos actuales, es fundamentalmente porque la filosofía tiene una pretensión de verdad que le asemeja a la ciencia más que al arte. Hay otras voces, de hecho, que argumentan que la situación actual de la filosofía debería ser distinta y defienden que los departamentos de filosofía dedican demasiado tiempo a la historia de la filosofía y demasiado poco a la filosofía como tal. En nombre de una visión más cientificista, unos rechazan esta presentación histórica de la filosofía, haciendo valer que al leer textos antiguos, uno no aprende su oficio sino más bien las malas manías de los pensadores antiguos y se arriesga si no a adoptar posiciones dogmáticas, por lo menos a hacer un uso indebido del argumento de autoridad. Es un poco lo que se ha defendido en Harvard en la época de Quine -que quiso prohibir las clases de historia- o, más recientemente, en Princeton, donde un profesor de filosofía había colgado un cartel diciendo "Just say no to the history of philosophy!", haciendo eco al "Just say no to drugs!" de Nancy Reagan (Cottingham, 2005, p. 25; Sorell, 2005, pp. 43-44). Aquí el modelo de historia en vigor sería más bien el que hemos caracterizado como el técnico-científico. No se niega por supuesto que la filosofía tenga una historia, pero se considera que su inscripción en la historia no es esencial en la medida en que, al igual que en las ciencias y en las técnicas, esa historia es vindicatoria, es decir, que nuevas teorías invalidan teorías antiguas a la vez que explican el modo de ver propio de las teorías anteriores. Según este punto de vista, la relevancia histórica innegable de, por ejemplo, Kant no afectaría directamente a la práctica actual de la filosofía. Aunque sea considerado uno de los mayores filósofos de la historia, no haber leído sus textos no importaría tanto porque sus logros, sean los que sean, habrían sido integrados al conocimiento filosófico general, como las leyes del movimiento de Newton son integradas a las leyes mecánicas actuales.

Esta concepción cientificista del saber filosófico tampoco consigue la unanimidad. Pero nos obliga a plantear la pregunta siguiente: ¿cómo se puede justificar que tenga importancia y que nos siga afectando un filósofo que ha pensado y ha escrito hace doscientos, cuatrocientos o hace 2500 años y cuyas verdades y doctrinas, sin embargo, no son recibidas por nadie como conocimientos asegurados? En la medida en que, como lo hemos señalado, no hay consenso sobre este problema fundamental, hay que reconocer la importancia de la cuestión sobre la relación de la filosofía presente con la tradición. 


\section{Historia de la historia de la filosofía}

Como es bien sabido, la actitud de muchos grandes filósofos frente a la tradición intelectual que les ha precedido ha sido de rechazo o de vigilancia. En cuanto investigación crítica o radical, la filosofía tiene como responsabilidad máxima la de sondear los presupuestos de cualquier argumento, que éste le venga dado por un pensador ilustre o no. El mismísimo Sócrates puede servir aquí de modelo de un pensador que, en vez de apoyarse sobre una tradición, exigía que se siguiera un argumento hasta donde llevara, con independencia de si el resultado de la investigación fuese conveniente o no (Cottingham, 2005, 26). Puestos a elegir entre lo que uno consigue demostrar y lo que heredamos de la tradición, es el deber de la filosofía rechazar la autoridad pasada en beneficio de la investigación racional. Esa voluntad "socrática" que consideramos como propia de la ilustración y de su rechazo a la tradición, se hizo patente en pensadores como Galileo y también como Descartes quien, relegando a Platón y a Aristóteles al museo, entregó la interrogación filosófica a la razón humana. Más cercano a nosotros en el tiempo, Edmund Husserl también adoptó esa actitud renacentista cuando, en 1900, publicó sus Invesigaciones lógicas cuyo lema "¡a las cosas mismas!" (Zurück zu den Sachen selbst!) se presentaba, en un principio, como un ataque en contra de todo tipo de tradicionalismos.

Sin embargo, esta actitud no es la única que haya existido a lo largo de la historia, ni siquiera la más extendida puesto que la filosofía no siempre ha querido guardar este tipo de distancias con la tradición. Además de ser un filósofo revolucionario, Aristóteles fue un gran y quizás el primer historiador de la filosofía. Siempre tiene ese reflejo muy hermenéutico de empezar la presentación de sus teorías por un retrato histórico del problema abordado y siempre se preocupa por mostrar cómo sus propuestas constituyen también una respuesta a las aporías de sus predecesores6. Del mismo modo, toda la filosofía de la antigüedad tardía y de la edad media sólo se puede entender en el marco de un diálogo constante con el pensamiento de Platón y Aristóteles. Y a partir del renacimiento y en la filosofía moderna, no hay unanimidad. Leibniz, un cartesiano de pura cepa, opone al método cartesiano lo que él llama su método histórico, un método que permita dar cuenta de la perennidad de los problemas filosóficos 7 . Pero la oposición entre las dos actitudes se hace más clara a partir del desarrollo de la conciencia histórica al final de la ilustración y de los textos de Herder -y en menor medida de Kant- sobre filosofía de la historia. En ese momento, la filosofía no tomó solamente conciencia de que otros pensadores habían pensado antes que ella, sino de algo mucho más importante: que su búsqueda de la verdad está inscrita en un largo proceso histórico que acaba condicionando

\footnotetext{
6 Véanse, por ejemplo, Metafisica A 3-6, De anima A 2 y Física A 2-4.

${ }^{7}$ Carta de Leibniz a Malebranche del 22 de junio de 1679, citada en Braun (1973).
} 
no sólo algunas de sus convicciones, sino también sus metas y los métodos para alcanzarlas. La mayor expresión de la conciencia histórica la encontramos en Hegel, para quien el proceso histórico de formación de la filosofía es a la vez el proceso de formación de la verdad que la filosofía está buscando. Pero como muchas veces se ha dicho, la muerte de Hegel en 1831 coincidió también con la muerte del idealismo alemán y con el nacimiento del positivismo, un movimiento filosófico y científico que, a pesar de nacer de una reflexión sobre la historia del pensamiento (en Auguste Comte), renovó la actitud de rechazo frente a la tradición filosófica.

Así, no podemos decir que la filosofía sea de por sí un rechazo a la tradición. La filosofía, a lo largo de toda su historia, ha usado las dos vías. Sin embargo, lo que une a esas dos formas de hacer filosofía es un rechazo claro al dogmatismo. Para muchos, la mejor forma para conseguirlo es rechazar en bloque lo que nos viene dado por la tradición. Para otros, sin embargo, uno no puede librarse del dogmatismo si no entiende bien este pasado que está condicionando su forma de hacer filosofía. Rechazar el pasado sería desde esta perspectiva la mejor forma de caer ciegamente en los prejuicios tradicionales.

\section{Filosofía analítica}

Aunque a menudo la filosofía analítica se presente a sí misma como la filosofía ahistórica por excelencia -a veces ella misma presenta su falta de interés por la historia de la filosofía como una de sus grandes calidades-, sería erróneo creer que esta situación sea hoy generalizada. Esta actitud de rechazo caracteriza sin duda al empirismo lógico del Círculo de Viena -Schlick, Neurath o Carnap- y quizás a Wittgenstein, quien, considerando que la filosofía no precisa considerar la historia, abre sin embargo sus Investigaciones filosóficas con un comentario de Agustín.

Aunque ese desinterés por la historia de la filosofía sea mayoritario, existen eminentes excepciones en el mundo analítico como los libros de Strawson o de Bennett sobre la Crítica de la razón pura de Kant (Strawson, 1966; Bennett, 1966) que han representado una renovación de los estudios kantianos en la filosofía analítica. Pero Kant no es el único filósofo histórico que interesa a los filósofos analíticos. De hecho, los grandes pensadores modernos - Descartes, Leibniz, Hobbes, Berkeley y Hume - aparecen a menudo en debates sobre temas filosóficos actuales, si bien es cierto que la mayoría de las veces lo hacen como "etiquetas" para indicar una solución clásica y errónea al problema.

También podemos subrayar que algunos hablan ahora de un cierto giro histórico en la filosofía analítica en la medida en que, desde los años 1970 o 1980, un cierto interés por la historia de la filosofía parece haberse despertado (Mulligan, 1997). Los Frege, Russel y Moore han terminado convirtiéndose en figuras históricas de la 
filosofía y leer sus textos hoy no supone sólo debatir las tesis que avanzan, sino también intentar comprender lo que querían decir, o sea interpretar sus textos. De este modo, la filosofía analítica llega a pronunciar juicios sobre su fundamento, sobre sus realizaciones y su progreso, aunque haya que reconocer que es sobre todo el caso de autores que hacen una crítica interna como son Richard Rorty y Hilary Putnam (Engel, 1997).

Pero, ¿qué método adopta la filosofía analítica a la hora de acercarse al pasado de la filosofía? Dejaremos aquí de lado el problema más específico de los métodos históricos que usan los historiadores que puedan provenir de la tradición analítica8. En filosofía, lo que podemos llamar el "ideal analítico en historia de la filosofía" (Janaway, 1988; Engel, 1999) consiste en leer a los filósofos del pasado como si fuesen nuestros contemporáneos. Como escribía Jonathan Barnes: "Hay que leer a Platón (...) como si hubiese publicado sus diálogos en Clarendon Press; hay que leer los ensayos de Aristóteles como si se acabasen de publicar en el último fascículo de Mind" (Barnes, 1980, p. 706). Solamente así podría tener un filósofo del pasado una relevancia que sea verdaderamente filosófica y no puramente histórica. Este ideal analítico impone varias condiciones para que un diálogo filosófico con autores del pasado pueda tener lugar. En vez de intentar reconstituir la coherencia de un autor gracias a una interpretación histórica, el filósofo procura extraer los problemas filosóficos planteados y las tesis propuestas como soluciones. El valor de verdad de las proposiciones así como la fuerza de los argumentos empleados para expresar estas tesis son evaluados con nuestros criterios. Así, se puede ver si, por muy poco probable que parezca, un autor del pasado no tendría una respuesta a nuestros problemas o, por lo menos, pistas para nuevas soluciones. De este modo, cuando lo considera necesario, el filósofo analítico se permite corregir a los autores del pasado de la misma forma que corrige a sus colegas, es decir construyendo nuevos argumentos a favor o en contra de las proposiciones consideradas. De alguna forma, el filósofo no sólo se interesa por lo que ha dicho un autor del pasado, sino también -aquí yace la fuerza filosófica de esta interpretación- por lo que hubiera debido decir.

Este método le suele parecer una herejía a los historiadores de la filosofía. Según el punto de vista del historiador, la "traductibilidad" de una filosofía antigua en términos actuales siempre es problemática. El aislar tesis de sus contextos también lo es. El historiador de la filosofía que sólo pretende evaluar la coherencia de un sistema filosófico nunca introduce en su estudio cosas que el filósofo estudiado no haya dicho. Así se oponen dos preocupaciones muy distintas: por un lado, la exactitud histórica y, por otro, el cuidado por la verdad. Aquí conviene citar la famo-

\footnotetext{
8 Sobre la cuestión de cómo uno debe escribir la historia de la filosofía y de sus varias formas, véase Jakobsson (2005, pp. 59-65) que presenta una tipología teniendo en cuenta los principales intentos llevados a cabo en los últimos cincuenta años.
} 
sa frase de Quine que decía, irónicamente, que sólo existen dos tipos de personas que se interesan por la filosofía: los que se interesan por la filosofía y los que se interesan por la historia de la filosofía (MacIntyre, 1984, pp. 39-40).

Esta actitud "no contextualista"9 que adoptan los filósofos analíticos frente a los pensadores del pasado parece dejarles muy pocas oportunidades para ser relevantes. La actitud del historiador que busca, de alguna forma, la coherencia de las falsedades antiguas podría de hecho parecer más respetuosa hacia las grandes figuras de la historia. Quizás corra el riesgo de hablar de temas filosóficamente irrelevantes, pero por lo menos no es injusto hacia el pasado. Sin embargo, podemos muy bien argumentar justamente lo contrario. Considerar que un filósofo que ha vivido hace siglos puede hoy todavía tener algo que decir en la filosofía contemporánea es el mayor o el más auténtico homenaje que se le pueda rendir (Granger, 1997, 123). Es lo que pasa ahora con varios autores medievales -por ejemplo, Guillermo de Ockham- que han entrado en debates sobre lógica, no como meras figuras históricas sino como filósofos en el sentido pleno de la palabra (Geach, 1980). Aunque se le hayan reprochado sus imprecisiones debidas a su falta de conocimiento histórico, P. T. Geach defiende que la relación del lógico frente a autores medievales tiene que entenderse de la misma forma que la de los matemáticos con los matemáticos antiguos:

Si le fuese prohibido a un lógico contemporáneo, en razón de su falta de Weltanschauung medieval, tratar de un problema lógico [medieval], entonces los algebristas contemporáneos deberían encontrarse igualmente en la imposibilidad de discutir los problemas de Diofante. (Geach, 1980, p. 9).

Aunque ya no seamos pensadores medievos, este hecho no debería impedirnos entrar en debate con ellos, puesto que suponemos que la filosofía trata de describir más o menos correctamente una realidad que no cambia a lo largo de la historia. Si los filósofos de antaño hubiesen vivido en mundos distintos al nuestro o si hubiesen tenido principios racionales diferentes, no podríamos entender sus tratados. Lo único que nos quedaría sería intentar una reconstrucción histórica de sus pensamientos (como con los jeroglíficos egipcios, por ejemplo). Pero no es lo que experimentamos al leer filosofía clásica. A pesar de las dificultades que puedan contener ciertos textos, somos capaces de seguir los argumentos y entender a qué se refieren los autores.

Así, existe en la filosofía analítica una convicción según la cual los problemas filosóficos que se plantearon en el pasado son en gran medida los mismos que se

\footnotetext{
9 Entendemos aquí por "contexto" las condiciones históricas, sociales, políticas, culturales, filosóficas etc. en las cuales aparece un pensamiento filosófico, condiciones que trascienden la mera estructura lógica de dicho pensamiento.
} 
plantean hoy o que existe un "espacio de problemas" en el cual se mueven los filósofos (Mulligan, 1997, pp. 64-65). Así como en la Problemgeschichte neokantiana de Windelband y de Rickert, la filosofía analítica tiene la convicción de que toda la tradición se ha confrontado con un número de problemas idénticos y que la historia del pensamiento puede ser leída como una serie de respuestas a estos problemas "eternos". Para Wittgenstein, la identidad de los problemas no es ningún misterio ya que se explica por la identidad del lenguaje que nos conduce siempre hacia las mismas preguntas. En un manuscrito fechado en 1933, Wittgenstein escribe:

nuestro lenguaje se ha mantenido igual [desde los griegos] y siempre nos conduce a las mismas preguntas. Mientras haya un verbo 'ser' que parece funcionar como 'comer' y 'beber', mientras haya adjetivos como 'idéntico', 'verdadero', 'falso', 'posible', mientras se hable del paso del tiempo y de la extensión del espacio, etc., etc., los hombres siempre volverán a toparse con las mismas dificultades enigmáticas y mirarán hacia algo que ninguna explicación parece conseguir eliminar. (Wittgenstein, 2005, p. 424).

Así, si los problemas filosóficos siempre han sido los mismos, podemos perfectamente imaginar debates entre filósofos de épocas distintas. De este modo, la filosofía analítica no establecería una ruptura con el pasado, sino que se inscribiría más bien en continuidad con la tradición y sus preguntas sobre el realismo, la verdad y la existencia. Si rompe con ella, es por su modo de preguntar y su formulación de dichas preguntas.

Entonces, al no colocar a Ockham en su contexto histórico, quizás faltemos a la exactitud histórica, pero podríamos estar rindiendo homenaje a su exactitud filosófica, es decir a su sentido de lo que importa en filosofía. También podría afirmar Geach que lo que le interesa son las tesis que encontró, de forma legítima o no, en la obra de Ockham. Así, no importaría tanto que los historiadores encuentren dichas tesis en la obra, en la medida en que Geach quiere debatir con tesis filosóficas y no con Ockham.

Así, vemos con claridad que lo que pretende la filosofía analítica cuando se acerca a la historia de la filosofía no es tanto un análisis de pensadores o de obras, sino más bien un análisis de las ideas. De esta forma, también escapa del culto rendido al genio que a menudo se encuentra en la filosofía continental. Al analítico le interesan ideas con las que debatir, sin importarle demasiado que esas hayan pertenecido a Kant, a Ockham o a un filósofo desconocido.

\section{Filosofía continental}

Cuando la filosofía analítica se acerca de forma filosófica a la historia de la filosofía, nunca o casi nunca lo hace renegando de su ahistoricismo (Engel, 1997). 
Aunque se interese por la historia, nunca intenta contextualizar su propia búsqueda de la verdad. Al contrario, suele mirar hacia ella para ver si la historia no podría ayudarla a solucionar problemas ya formulados en presente. No está poniendo en duda la legitimidad de sus problemas a partir de un estudio histórico. Sin embargo, tiene que reconocer, aunque sea de forma mínima, que está ligada a la tradición filosófica. Como hemos visto citando a Wittgenstein, él considera que hay mucho en común entre los problemas actuales y los de antes, no tanto porque sean los que se plantean de forma natural en el mundo donde vivimos, sino más bien porque son los que surgen de un lenguaje compartido. Así, aunque se quiera romper con todas las soluciones del pasado, heredamos implícitamente una serie de problemas y de conceptos que tienen su origen en el pasado.

Un "grado cero" de historicidad, si me puedo expresar así, es una utopía difícil de defender. El mero hecho de llamar "filosofía" a lo que seguimos haciendo hoy supone que encontramos suficientes paralelismos entre nuestra tarea actual y lo que instauró Platón en el siglo cuarto antes de Cristo. Toda actividad filosófica, explícita o implícitamente, se inscribe en la continuidad de una tradición y trabaja con problemas y conceptos en su mayor parte heredados. Los conceptos de "libertad", "mundo", "existencia", "posibilidad", "definición", "cuerpo" "sujeto", "objeto" son todos conceptos que tienen un pasado, que están "cargados" de tradición y cuyos sentidos han sido fijados por pensadores pertenecientes al pasado. Para muchos pensadores, la mayoría europeos, esto significa que, de un modo u otro, la filosofía pasada quizás no determina pero sí condiciona nuestra forma de filosofar hoy ${ }^{10}$. En la perspectiva analítica, se puede considerar que la "ignorancia histórica" es justamente un arma contra este condicionamiento que afectaría a los que se han nutrido de la filosofía del pasado. Pero la pregunta que muchos filósofos se hacen en el continente es justamente saber si tal rechazo no hace más que crear la ilusión de que hemos vuelto al principio de la filosofía y que podemos por fin filosofar en plena libertad. De este lado del Canal de la Mancha, varios autores consideran que la retoma de los conceptos filosóficos tradicionales no puede llevarse a cabo sin la aceptación o la adopción implícita de decisiones históricas tomadas en el pasado.

Según este punto de vista, la actividad filosófica sería una tarea heredada $\mathrm{y}$, como resultado, los problemas filosóficos serían condicionados por un pasado filosófico que afecta todavía hoy nuestra práctica de la filosofía. La filosofía continental -o para ser más preciso, la hermenéutica- considera que a pesar de que haya sido demostrado que eran falsas, las filosofías no desaparecen como si hubiesen sido

\footnotetext{
10 En castellano como en francés o en inglés, hablar de la determinación del pensamiento filosófico puede hacer creer que nos referimos a una suerte de determinismo. Por eso hablaré de "condicionamiento" a pesar de que en esa palabra resuene desafortunadamente la condición (Bedingung) en vez de la determinación (Bestimmung). El problema no se presenta en alemán donde existe una distancia etimológica entre los conceptos de Bestimmtheit y de Determinismus.
} 
superadas o como formas "epistemológicamente monstruosas" (Michaud, 1997, p. 159) de hacer filosofía, tal y como ocurre en ciencias. Las filosofías pasadas son momentos de una historia que llega hasta nosotros, de una historia que explica porqué, por ejemplo, la realidad del mundo exterior nos pueda parecer problemática. Este punto de vista defiende que uno sólo puede descubrir lo que vale como problema en filosofía al leer la historia de la filosofía. Los problemas no se darían al filósofo de forma obvia - como a un ingeniero un río encima del cual construir un puente- sino que sería el fruto de un aprendizaje histórico.

Por eso, el filósofo continental siempre se preocupa por la historia de los conceptos que usa e intenta determinar hasta qué punto la retoma de los conceptos tradicionales supone la adopción implícita de ciertas posiciones o de cierto marco filosófico. El filósofo continental suele considerar la investigación histórica sobre los conceptos no como un adorno, sino como una tarea filosófica fundamental. El uso lúcido y transparente de los conceptos depende, según esta perspectiva, del conocimiento que uno tiene del origen de los conceptos y del camino histórico que tuvieron que recorrer para llegar hasta nosotros.

Esta forma de hacer filosofía, hay que reconocerlo, retrasa el progreso. También, no hay acuerdo ni debate sobre lo que buscan realmente tales exámenes históricos, es decir: ¿cuándo un concepto alcanza la transparencia, si algún día la alcanza? ¿Hasta dónde en el tiempo tenemos que retroceder (Descartes, los griegos, los presocráticos, el orfismo, etc.)? Para muchos críticos, la filosofía se transforma entonces en una tarea filológica que, al fin y al cabo, tiene poco que ver con lo que la filosofía quería ser. Y sobran ejemplos para demostrarlo. Herbert Schnädelbach critica la tradición hermenéutica hablando de una "Philologisierung der Philosophie" (Schnädelbach, 1987, p. 279), transformándola en un trabajo histórico que ya no es filosofía.

Pero aunque la hermenéutica se haga desde un gran respeto por la historia de la filosofía, ella también se enfrenta al problema de la exactitud histórica. Las grandes interpretaciones de la historia de la filosofía que se han presentado en la filosofía continental desde Heidegger hasta Alasdair MacIntyre, pasando por Blumenberg o Foucault, reciben a menudo críticas de los historiadores "oficiales" de la filosofía. Aquí cabe decir que este problema tiene mayores implicaciones que en la tradición analítica. Como lo hemos subrayado, para la filosofía analítica no importa en el fondo que se esté hablando de las verdaderas tesis de Guillermo de Ockham o de algunas tesis que se defienden o se rechazan. Pero si nos apoyamos en la idea de que la historia de la filosofía condiciona nuestra posición filosófica actual, la cuestión de la exactitud histórica cobra mucha importancia. Si se considera que el pensamiento de Kant nos sigue determinando después de doscientos años y si se busca de qué forma lo está haciendo, no podemos inventarnos un Kant que no tenga que ver con el auténtico. Sin embargo, hay que mencionar que la influencia que un pen- 
sador puede seguir teniendo, a menudo no es tanto cosa suya como cosa de la tradición. La historia de las influencias a veces puede decir más que el estudio histórico de un pensador. Así, el Kant o el Descartes que condiciona nuestras prácticas filosóficas, no tiene por qué ser el auténtico Kant o Descartes, sino que a menudo es el resultado del auténtico pensador y de lo que se llama en hermenéutica la "historia efectual" o el "trabajo de la historia" (Wirkungsgeschichte). En ese sentido, cabe hablar en historia de la filosofía, al igual que en historia del arte, de cánones y de clásicos, que se refieren sin duda más a la recepción de las obras que a las obras mismas. Con Descartes, me parece obvio: el Descartes que la tradición moderna ha conservado es un pensador racional que fundamenta de nuevo la ciencia sobre la certeza de su propio existir (Meditaciones primera y segunda). Pero el proyecto del Descartes "real" no era otro que el de demostrar la existencia de dios y la distinción real entre el cuerpo y el alma (Meditación tercera). Las dos primeras meditaciones cobran una importancia mucho mayor que la tercera, no tanto por el proyecto que Descartes quería llevar a cabo, sino por el rumbo que siguió la historia del pensamiento en la época moderna.

Existen, pues, varios matices y varios debates posibles sobre la exactitud histórica y sobre si la historia de la filosofía debe ser escrita por historiadores o por filósofos. Pero lo que cabe subrayar aquí, es que los filósofos tanto analíticos como continentales reciben críticas de los historiadores oficiales. Cuando Strawson, en The Bounds of Sense, se acerca a la obra de Kant y la interpreta a partir de los estándares filosóficos de hoy, los historiadores dicen que tal cosa es imposible, que uno no puede leer a Kant sin tener en consideración el proyecto propio de la Crítica de la razón pura que es el de una reforma del pensamiento metafísico racional. Los historiadores consideran que el trabajo de Strawson quizás valga para arrojar luz sobre el pensamiento de Strawson pero que su crítica no toca a Kant en absoluto (Hatfield, 2005, pp. 94-97). Lo curioso es que lo mismo pasa con la obra de Heidegger sobre la Crítica de la razón pura, Kant und das Problem der Metaphysik. En esta obra, Heidegger intenta mostrar que Kant se acercó en la Crítica de la razón pura al proyecto ontológico que expuso en Ser y tiempo. Para defender esa tesis, Heidegger se apoya en lo que, según él, Kant quería pero no supo decir (Heidegger, 1977, p. 3). Como en el caso de Strawson, los historiadores subrayan que lo que dice Heidegger es históricamente inexacto en la medida en que no se encuentra en el texto de Kant. En ambos casos, podríamos sostener que al lado de la verdad filosófica, el problema de la exactitud histórica es un problema secundario. Sin embargo, este problema de la exactitud histórica quizás revele algo que tenga relevancia filosófica. 


\section{El problema de la exactitud histórica}

El problema que trae consigo el dialogo histórico es el siguiente: ¿podemos dialogar con Kant sin tener en cuenta la distancia histórica que nos separa de él o sin tener en cuenta el contexto que dio sentido a sus afirmaciones? Desde la perspectiva analítica, no tendría por qué ser problemático puesto que uno debate con tesis cuya validez se puede comprobar lógicamente sin tener en cuenta la más mínima condición histórica. Si encontramos en Kant una afirmación que constituye una falsedad lógica, ¿de qué forma el contexto histórico podría salvarla? Las circunstancias atenuantes en filosofía no parecen tener que interesarnos.

Sin embargo, para poder dialogar con filósofos del pasado, no sólo debemos suponer que compartimos mundo y principios racionales básicos, como hemos dicho antes, sino también problemas. La hipótesis básica del diálogo anacrónico es la constancia de los problemas filosóficos. Tal visión de la historia de la filosofía es la que defendían los neo-kantianos con su "historia de los problemas" (Problemgeschichte) oponiéndose a la lectura hegeliana de la historia de la filosofía, en la cual cada etapa de la historia alcanza nuevos problemas cada vez más complejos. Los neo-kantianos consideraban la filosofía como una larga serie de respuestas dadas a preguntas idénticas (Windelband, 1980, p. iv; Hartmann, 1910, pp. 465466). Pero esta concepción de la historia de la filosofía tiene fallos. Cuando uno se interesa desde un punto de vista histórico por la historia de la filosofía, descubre que existen inmensas diferencias entre lo que en un principio parecen ser problemas idénticos. No es tanto que la forma de solucionar los problemas haya cambiado, sino más bien que la forma de entender la filosofía, la forma de definir sus tareas y de formular los problemas tienen a veces poco que ver con las nuestras. Como escribe Daniel Garber,

Se supone a menudo que la disciplina "filosofía" que practicamos hoy en día es sustancialmente la misma que fue en tiempos pasados. Esta suposición es la que está en el fundamento de la manera en que los filósofos han usado la historia de la filosofía como una fuente de argumentos y de problemas para sus trabajos presentes. Pero un estudio cuidadoso y auténticamente histórico de la filosofía moderna temprana nos da una idea muy distinta del tema, algo que nos puede enseñar cosas a los filósofos del siglo veintiuno. (Garber, 2005, p. 138).

Tomando a Descartes como ejemplo, Garber muestra con precisión cómo a pesar de presentar similitudes, el problema del escepticismo o el de la libertad de la voluntad en Descartes no se corresponden a los nuestros (Garber, 2005, pp. 138140). Aunque las formulaciones puedan parecerse, lo que está en juego es tan radicalmente distinto y está ligado a un desafío tan alejado que no podríamos comparar las soluciones obviando el contexto y sobre todo, los "proyectos filosóficos" propios de cada pensador (Hatfield, 2005, pp. 103-104) o las "imágenes filosóficas" que cada uno quiere promover (Piercey, 2009, pp. 19-25). 
Quizás entender la posición que ocupamos dentro de la historia puede, al final, darnos una mejor idea de cómo se articula nuestra tarea filosófica. Subrayar la naturaleza histórica de la interrogación filosófica y considerarnos a nosotros mismos como "figuras históricas" nos puede permitir tomar conciencia de que nuestras prácticas filosóficas no son tan transparentes como lo pueden parecer. Obviamente, esto podría llevarnos a adoptar la posición muy poco envidiable del relativismo histórico. Aquí yace el mayor desafío para la filosofía que quiera entenderse de forma histórica: encontrar un punto medio entre el antihistoricismo cientificista y el relativismo histórico.

Un ejemplo que, a mi modo de ver, es interesante al respecto es el de la práctica actual de la ética. Podemos considerar que hubo una revolución enorme en ética con la aparición del utilitarismo de John Stuart Mill a mitad del siglo diecinueve. Aunque podamos defender que las teorías utilitaristas han perdido en los últimos años el prestigio del que gozaron en otras épocas, la forma de entender los problemas éticos propia del utilitarismo sigue vigente. En efecto, la ética se ha transformado después de Kant en un intento de encontrar la fórmula que más acierta en la resolución de los problemas que plantea la vida ética. Comparando los debates actuales con los escritos de Kant, uno se da cuenta de que el filósofo de Königsberg no tenía en absoluto intención de ofrecer fórmulas para resolver problemas éticos. En la Fundación de la metafísica de las costumbres, Kant intenta más bien descubrir los "mecanismos" racionales situados detrás de las acciones morales. Ahí descubre, como es bien sabido, el imperativo moral y la autonomía de la voluntad. Pero cuando Kant habla del imperativo moral, no lo presenta como un truco infalible para saber cómo actuar en situaciones precisas, sino como la ley que ya está actuando desde siempre en nuestra razón práctica. Lo que pretende describir Kant es cómo está funcionando la vida del sujeto moral, una tarea que al fin y al cabo está ausente de los debates éticos actuales.

Así, a pesar de rechazar la solución del utilitarismo, hemos cambiado el problema ético kantiano por el de John Stuart Mill y la práctica kantiana por la utilitarista. A mi modo de ver, toda la ética actual está condicionada por esa idea utilitarista según la cual la meta de la ética es la de encontrar una fórmula que pueda ser aplicada de forma universal a los problemas éticos que se nos plantean. Hasta John Rawls, un kantiano, no hace más que buscar una forma que nos permita solucionar los problemas morales o políticos que se nos presenta. Plantear la pregunta ética "¿qué debemos hacer?” no tiene por que significar “¿cuál es la fórmula universal que me permite salir de cualquier problema ético?" y la obra de Kant nos lo muestra magistralmente.

Aquí tenemos un ejemplo de una teoría filosófica que ya no recibe el asentimiento general y que, sin embargo, ha impuesto un marco de problematización que ha transformado las prácticas. Reflexionar sobre la historia de la ética nos puede 
servir, como mencioné antes, para esclarecer nuestra forma de hacer filosofía y ponerla en perspectiva. La historia de la filosofía es, en ese sentido, la mejor manera -quizás la única manera- de interrogar nuestra práctica filosófica y transformarla si es necesario.

Estas ideas son defendidas hoy por varios pensadores como Bernard Williams o Charles Taylor. Según éste último, el uso de la historia de la filosofía es el único medio que tenemos para escapar del dogmatismo que siempre amenaza a la filosofía. Según Taylor, la hegemonía del modelo epistemológico en la filosofía actual -que Taylor asocia con Quine y al que se opone- no puede ser desmantelado desde dentro. Para Taylor, ese modelo tiene muchos fallos y eso sólo se puede ver cuando uno vuelve a las raíces históricas del modelo epistemológico, o sea a Descartes y a Kant. Es preciso volver a Descartes por medio de lo que Taylor llama una "consideración genética" (genetic account) y así poner en evidencia los modelos concurrentes al modelo matemático cartesiano (Taylor, 1984, pp. 18-20). Es lo que pretende llevar a cabo en su obra Sources of the Self (Taylor, 1989), mostrando cómo el modelo de un sujeto histórica y culturalmente desligado (disengaged) es una utopía que funciona en la filosofía actual como un prejuicio.

Para entender adecuadamente quiénes somos, defiende Taylor, hay que entender cómo hemos llegado donde estamos (Taylor, 1984, 28). Volviendo a Descartes, Taylor pretende mostrar que existían entonces otros modelos que hubieran podido ser adoptados, como el modelo humanista. Pero se pueden aportar otros argumentos a favor de la naturaleza histórica de la filosofía. Inspirándose en Nietzsche, Bernard Williams sostenía que la lectura de los grandes filósofos permite algo extraordinario: "hacer que lo habitual (the familiar) parezca extraño" (Williams, 1994, p. 21). Trasformando los temas de nuestros debates en algo digno de ser interrogado, la historia nos obliga a detenernos y a volver a evaluar nuestras evidencias y nuestras suposiciones. Los filósofos que no disponen de esta perspectiva histórica se convierten en "prisioneros del presente" (MacIntyre, 1984, 33) o sucumben a la "tiranía del presente" (Garber, 2005, 145).

\section{Conclusión}

La filosofía, al fin y al cabo, no puede adoptar ninguno de los modelos de historia que hemos presentado en un primer momento. No se adapta al modelo de la ciencia porque no tiene el "privilegio" de tener que contar con las limitaciones que el mundo natural le impone. Mientras el científico "se conforma con las restricciones de la lógica y la piedra de toque de la evidencia empírica" (Cottingham, 2005, p. 28), el filósofo puede reescribir las leyes lógicas y puede debatir de temas cuya existencia se quede en el ámbito de lo posible. Así, el modelo cientificista de algu- 
nos filósofos fuerza la filosofía a adoptar una forma de historia que no le es natural: una historia vindicatoria que contaría con logros indiscutibles ${ }^{11}$.

Sin embargo, el modelo histórico del arte tampoco se ajusta a la historia de la filosofía. Aunque podamos defender que las exigencias actuales ligadas a la tarea filosófica son heredadas históricamente al igual que en el arte, es arriesgado comparar la búsqueda filosófica de lo verdadero con la búsqueda propia del arte. No sólo porque el arte ya no le es fiel a la búsqueda de lo bello, sino más bien porque la voluntad de ruptura con el pasado y la necesidad permanente de innovar propia del mundo del arte no es nada filosófica. La filosofía se pararía de inmediato si encontrase soluciones absolutas a sus problemas, mientras que el arte no contempla la idea de una adecuación satisfactoria.

Podemos pues defender que la filosofía tiene en común con el arte el condicionamiento por parte de su historia y con la ciencia, el contemplar la posibilidad de dar una respuesta correcta a sus preguntas. Así, hay que pensar en un tercer modelo de historia que esté a mitad camino entre el del arte y el de la ciencia. Es lo que quería defender hablando de encontrar un punto medio entre el antihistoricismo cientificista y el relativismo histórico. El problema que plantea tal modelo es obviamente el de hacer compatibles el condicionamiento histórico con la posibilidad de una verdad no relativa.

En cuanto a la relación que el filósofo actual debe mantener con sus antepasados puede tomar como modelo tanto a las artes como a las ciencias. El filósofo nunca lee a los clásicos de la filosofía con los ojos de un historiador desinteresado. La búsqueda de la verdad y la posibilidad de adecuarse a la experiencia humana presentes en esas obras es lo que verdaderamente le importa. Sin embargo, algunos de estos textos sólo se entienden cuando uno los contextualiza, es decir cuando uno toma conciencia de que la comprensión humana del mundo no ha sido siempre la misma aunque el mundo mismo no haya cambiado. Así, leyendo a los griegos o a los modernos nos podemos topar con experiencias que nos desestabilizan, que nos permiten dar cuenta de que nuestras evidencias no son evidencias universales y que lo que en un principio nos parecen ser errores por falta de conocimiento son en realidad perspectivas diferentes y tan valiosas como las nuestras sobre el mundo. De esta forma, no se lee a los autores antiguos como si acabasen de publicar en Mind, sino como siguiendo pautas que nos son menos conocidas pero que tienen su valor propio y que pueden arrojar una luz inquietante pero lúcida sobre nuestro presente filosófico. Como escribía Bernard Williams, intentar borrar la distancia que nos

11 Puede sorprender que algunos estén hoy en día dispuestos a hablar de "resultados definitivos" en filosofía como si de la ley de la conservación de la materia se tratara. No duda en hacerlo Tom Sorell (2005, pp. 50-51) refiriéndose a la teoría de las "descripciones" de Russell y a la del "designador rígido" de Kripke. Pero, ¿sobre la base de qué criterios uno puede asegurar que esas teorías son definitivas para el resto de la historia? 
separa de los autores antiguos para leerlos como contemporáneos sólo consigue una cosa: "aniquilar el principal interés filosófico que pueda tener leer a [filósofos antiguos]" (Williams, 2000, p. 478). Aquí también, hay que encontrar un punto medio entre la lectura puramente histórica de la filosofía antigua y la lectura "contemporaneizante" que traduce en conceptos actuales los problemas antiguos ${ }^{12}$. Interpretar a los clásicos de la filosofía como si hubiesen querido dar una respuesta a nuestras formulaciones de los problemas filosóficos no sólo es injusto, revela también una superficialidad histórica que casa mal con la tarea filosófica como tal.

Para que la lectura de los filósofos antiguos sea de provecho, no sólo a nivel cultural sino también filosófico, esa lectura tiene que transformarnos, al igual que los viajes por el mundo modifican la percepción que uno tiene de su propio mundo. Es lo que Descartes defendía en la primera parte del Discurso del método donde escribe: "Pues es casi lo mismo conversar con gente de otros siglos que viajar. Bueno es saber algo de las costumbres de otros pueblos para juzgar las del propio con mejor acierto" (AT VI, 6). En un mundo donde todos los humanos pasarían sus días respondiendo a las mismas exigencias vitales, viajar sólo vendría a confirmar lo que ya sabemos.

\section{Referencias bibliográficas}

BARneS, J. (1980): "Aristote chez les Anglophones », en Critique, 399-400, pp. 704-718.

Bennett, J. (1966): Kant's Analytic, Cambridge, Cambridge University Press.

Boss, G. (ed.) (1994): La philosophie et son histoire, Zurich, Grand Midi.

Braun, L. (1973): Histoire de l'histoire de la philosophie, Paris, Ophrys.

Collingwood, R. G. (1983): An Autobiography, Oxford, Clarendon Press.

Cottingham, J. (2005): "Why Should Analytic Philosophers Do History of Philosophy?", en T. Sorell y G. A. J. Rogers (eds.), Analytic Philosophy and History of Philosophy, Oxford, Clarendon Press, pp. 25-41.

Courtine, J.-F. (ed.) (1999): La philosophie et ses histoires. Les Études philosophiques, 4.

D’Agostini, F. (1997): Analitici e continentali. Guida alla filosofia degli ultimi trent'anni, Milano, R. Cortina.

EnGEL, P. (1999): "Retour aval”, en Les études philosophiques, 4, pp. 453-463.

ENGEL, P. (1997): "La philosophie analytique doit-elle prendre un tournant histori-

12 Es lo que hacían, a ojos de Collingwood, los viejos realistas que insistían en traducir a los griegos en conceptos actuales. Pero, como lo relata, si insistimos en traducir el griego "trieme" por "buque a vapor", es muy probable que lleguemos a la conclusión de que los griegos tenían un concepto defectuoso del buque a vapor (Collingwood, 1983, p. 63) 
que ?", en J.-M. Vienne (ed.), Philosophie analytique et histoire de la philosophie, París, Vrin, pp. 139-152.

Garber, D. (2005): "What's Philosophical about the History of Philosophy?", en T.

Sorell and G. A. J. Rogers (eds.), Analytic Philosophy and History of Philosophy, Oxford, Clarendon Press, pp. 129-146.

Geach, P. T. (1980): Reference and Generality, Ithaca, Cornell University Press.

Gracia, J. J. E. (1992): Philosophy and its History. Issues in Philosophical Historiography, New York, SUNY.

Granger, G. G. (1997): “Analyse des oeuvres, analyse d'idées", en J.-M. Vienne (ed.), Philosophie analytique et histoire de la philosophie, París, Vrin, pp. 121125.

Grondin, J. (2000): "Continental or Hermeneutical Philosophy. The Tragedies of Understanding in the Analytic and Continental Perspectives", en C. E. Scott y J. Sallis (eds.), Interrogating the Tradition: Hermeneutics and the Histroy of Philosophy, New York, SUNY, pp. 75-83.

Hare, P. H. (ed.) (1988): Doing Philosophy Historically, Buffalo, Prometheus Books, 1988.

Hartmann, N. (1910): "Zur Methode der Philosophiegeschichte", en Kant-Studien, 15 , pp. 459-485.

Hatfield, G. (2005): "The History of Philosophy as History", en T. Sorell and G. A. J. Rogers (eds.), Analytic Philosophy and History of Philosophy, Oxford, Clarendon Press, pp. 83-128.

HeIDEgGeR, M. (1977): Phänomenologische Interpretation von Kants Kritik der reinen Vernunft, Frankfurt a. M., Kostermann.

Husserl, E. (1987): Aufsätze und Vorträge (1911-1921), Husserliana, tómo XXV, La Haya, Martinus Nijhoff.

JAKOBSSON, J. (2005): "Prolegomena to the historiography of Philosophy", en M. S. C. Schuback y H. Ruin (eds.), The Past's Presence. Essays on the Historicity of Philosophical Thinking, Huddinge, Södertörns högskola, pp. 55-92.

JANAWAY, C. (1988): "History of Philosophy: the Analytical Ideal", en Proceedings of the Aristotelian Society, LXII, pp. 169-189.

MacIntyre, A. (1984): "The Relationship of Philosophy to its Past", en R., Rorty, J. B. Schneewind y Q. Skinner (eds.), Philosophy in History. Essays on the Historiography of Philosophy, Cambridge University Press, pp. 31-48.

Michaud, Y. (1997): “La fin de l'histoire de la philosophie?", en J.-M. Vienne (ed.), Philosophie analytique et histoire de la philosophie, París, Vrin, pp. 153-163.

Mulligan, K. (1997): "Sur l'histoire de l'approche analytique de l'histoire de la philosophie (de Bolzano et Brentano à Bennett et Barnes)", en J.-M. Vienne (ed.), Philosophie analytique et histoire de la philosophie, París, Vrin, pp. 61103. 
Piercey, R. (2009): The Uses of the Past from Heidegger to Rorty. Doing Philosophy Historically, Cambridge University Press.

RÉE, J. (1997): "La philosophie anglaise des années cinquante", en J.-M. Vienne (ed.), Philosophie analytique et histoire de la philosophie, París, Vrin, pp. 1760.

RICKERT, H. (1915): Der Gegenstand der Erkenntnis. Einfuhrung in die Transzendental-Philosophie, Tübingen, Mohr.

Rorty, R., Schneewind, J. B. y Skinner, Q. (eds.) (1984), Philosophy in History. Essays on the Historiography of Philosophy, Cambridge University Press.

SÁEz Rueda, L. (2002): El conflicto entre continentales y analíticos. Dos tradiciones filosóficas, Barcelona, Crítica.

SÁNFEliX VidARTE, V. (2007): "El pasado de la filosofía y la formación filosófica", en Experientia et Sapientia. Estudios dedicados a la memoria de Ángel Álvarez Gómez, Universidade Santiago de Compostela, pp. 471-480.

SCHNÄDElBACH, H. (1987): "Morbus Hermeneuticus. Thesen über eine hermeneutische Krankheit", en Vernunft und Geschichte. Vorträge und Abhandlungen, Frankfurt am Main, Suhrkamp.

Schuback, M. S. C. y Ruin, H. (2005): The Past's Presence. Essays on the Historicity of Philosophical Thinking, Huddinge, Södertörns högskola.

Sorell, T., y Rogers, G. A. J. (eds.) (2005): Analytical Philosophy and History of Philosophy, Oxford, Clarendon Press.

Sorell, T. (2005): "On Saying No to the History of Philosophy", en T. Sorell y G. A. J. Rogers (eds.), Analytical Philosophy and History of Philosophy, Oxford, Clarendon Press, pp. 43-59.

Strawson, P. F. (1966): The Bounds of Sense. An Essay on Kant's Critique of Pure Reason, Londres, Routledge.

TAYLOR, C. (1989): Sources of the Self. The Making of the Modern Identity, Boston, Harvard University Press.

TAYlor, C. (1984): "Philosophy and its History," en Philosophy in History. Essays on the Historiography of Philosophy, R. Rorty, J. B. Schneewind y Q. Skinner (eds.), Cambridge University Press, pp.17-30.

VAtтimo, G. (ed.) (1989): Que peut faire la philosophie de son histoire?, París, Le Seuil.

VIEnNe, J.-M. (ed.) (1997): Philosophie analytique et histoire de la philosophie, París, Vrin.

Williams, B. (2000): "Philosophy as a Humanistic Discipline", en Philosophy, 75, pp. 477-496.

Williams, B. (1994): "Descartes and the Historiography of Philosophy", en J. Cottingham (ed.), Reason, Will and Sensation: Studies in Descartes' Metaphysics, Oxford, Clarendon Press, pp. 19-27. 
WindeLBAND, W. (1980): Lehrbuch der Geschichte der Philosophie (1892), Tuibingen, Mohr Siebeck.

Wittgentstein, L. (2005): The Big Typescript, TS-213, Bergen Text Edition/Oxford, Blackwell Publishing.

François Jaran

Departament de Metafísica i Teoria del Coneixement

Universitat de València

francois.jaran@uv.es 